\title{
Exact Relations between Multifractal Exponents at the Anderson Transition
}

\author{
A. D. Mirlin, ${ }^{1,2, *}$ Y. V. Fyodorov, ${ }^{3}$ A. Mildenberger, ${ }^{4}$ and F. Evers ${ }^{1,2}$ \\ ${ }^{1}$ Institut für Nanotechnologie, Forschungszentrum Karlsruhe, 76021 Karlsruhe, Germany \\ ${ }^{2}$ Institut für Theorie der kondensierten Materie, Universität Karlsruhe, 76128 Karlsruhe, Germany \\ ${ }^{3}$ School of Mathematical Sciences, University of Nottingham, Nottingham NG7 2RD, United Kingdom \\ ${ }^{4}$ Fakultät für Physik, Universität Karlsruhe, 76128 Karlsruhe, Germany
}

(Received 14 March 2006; published 28 July 2006)

\begin{abstract}
Two exact relations between mutlifractal exponents are shown to hold at the critical point of the Anderson localization transition. The first relation implies a symmetry of the multifractal spectrum linking the exponents with indices $q<1 / 2$ to those with $q>1 / 2$. The second relation connects the wavefunction multifractality to that of Wigner delay times in a system with a lead attached.
\end{abstract}

DOI: 10.1103/PhysRevLett.97.046803

Anderson localization transitions in disordered electronic systems (including both the localizationdelocalization transitions and the quantum Hall transitions between two phases with localized states) represent a remarkable class of quantum phase transitions. A hallmark of these transitions is the multifractality (MF) of electronic wave functions, describing their strong fluctuations at criticality [1]: the wave functions are characterized by a whole set of fractal dimensions $D_{q}$ different from the spatial dimensionality $d$. While at present the wavefunction MF is routinely observed only in computer simulations, rapidly developing imaging techniques allow to hope for its forthcoming experimental observation.

In this Letter we point out two exact relations satisfied by the multifractal dimensions. The first of these relations connects exponents with $q$ larger and smaller than $1 / 2$. The second relation links the multifractal indices for the wave functions of a closed system to those for the Wigner delay times characterizing the wave scattering from the same system via an attached lead.

We begin by considering a relation for the distribution function of the local density of states (LDOS) $\rho$,

$$
\mathcal{P}_{\rho}(\tilde{\rho})=\tilde{\rho}^{-3} \mathcal{P}_{\rho}\left(\tilde{\rho}^{-1}\right) .
$$

Here $\tilde{\rho}$ is the LDOS normalized to its average value, $\tilde{\rho}=$ $\rho /\langle\rho\rangle$ (the normalization factor is not critical and plays no role for our discussion). Introducing the LDOS moments, $\left\langle\tilde{\rho}^{q}\right\rangle=\int d \tilde{\rho} \tilde{\rho}^{q} \mathcal{P}_{\rho}(\tilde{\rho})$, we find that Eq. (1) implies the relation $\left\langle\tilde{\rho}^{q}\right\rangle=\left\langle\tilde{\rho}^{1-q}\right\rangle$. What is of central importance here is the status of Eq. (1). Specifically, this formula is exact on the level of the nonlinear $\sigma$ model (NL $\sigma \mathrm{M})$. It was derived for the first time in Ref. [2] for the case of systems with broken time-reversal invariance. The reasons for its general validity were revealed in Ref. [3], and an explicit derivation for all standard Wigner-Dyson symmetry classes was provided in Ref. [4].

Clearly, a mapping of a particular microscopic model of a disordered system (e.g., an electron in a random potential with certain correlation function) onto the NL $\sigma \mathrm{M}$ is not exact. More specifically, it is approximately valid in the
PACS numbers: 73.20.Fz, 05.45.Df, 72.15.Rn, 73.43.-f

case of weak disorder and breaks down for strong disorder. Therefore, the relation (1) has the same status. Nevertheless, we argue that a relation between the anomalous multifractal exponents $\Delta_{q}$ characterizing the behavior of the moments $\left\langle\rho^{q}\right\rangle$ at criticality,

$$
\Delta_{q}=\Delta_{1-q},
$$

which follows from (1), is exact.

Before explaining this, we digress with a brief reminder of the wave-function MF formalism; the reader is referred to the reviews [1] for a more detailed exposition. The moments of a wave function (so-called inverse participation ratios) $P_{q}=\int d^{d} r|\psi(\mathbf{r})|^{2 q}$ show at criticality an anomalous scaling with respect to the system size $L$,

$$
\begin{gathered}
\left\langle P_{q}\right\rangle=L^{d}\left\langle|\psi(\mathbf{r})|^{2 q}\right\rangle \propto L^{-\tau_{q}}, \\
\tau_{q} \equiv D_{q}(q-1)=d(q-1)+\Delta_{q} .
\end{gathered}
$$

Here $\Delta_{q}$ are anomalous exponents distinguishing a critical point from a metallic phase. These exponents also govern the scaling of the moments of LDOS,

$$
\left\langle\rho^{q}\right\rangle \propto L^{-\Delta_{q}} .
$$

Equivalently, the MF can be described by so-called singularity spectrum $f(\alpha)$, which is the Legendre transform of $\tau_{q}$. Its meaning is as follows: the average measure of a set of those points $\mathbf{r}$ in a sample, where the wave function behaves as $\left|\psi^{2}(\mathbf{r})\right| \sim L^{-\alpha}$, scales with $L$ as $L^{f(\alpha)}$.

We return now to the proof of exactness of Eq. (2). The central argument relies crucially on the universality of critical properties at the Anderson transition. Specifically, while the mapping of the original microscopic model onto the $\mathrm{NL} \sigma \mathrm{M}$ is at most approximate, one can find another microscopic model [e.g., $N$-orbital Wegner model in the limit $N \rightarrow \infty$ [5], which can also be viewed as a model of a granular metal [6]] that can be reduced exactly to the NL $\sigma \mathrm{M}$. The universality implies that the original microscopic model and the NL $\sigma \mathrm{M}$ must flow under renormalization-group transformations into the same fixed point in the infrared limit and will thus have the same 
critical exponents [governed by the RG properties in the vicinity of this fixed point; see, e.g., [7]]. Therefore, the relation (2) must hold not only in the NL $\sigma \mathrm{M}$ approximation, but be an intrinsic property of any generic microscopic model, even though the validity of Eq. (1) is in general only approximate. Remarkably, the universality allows us to prove an exact relation which does not follow from any simple scaling considerations.

The moments of the LDOS and of the wave-function intensity, which we considered above, are properties of a closed system. An alternative method to study the local properties is to open the system by attaching a perfectly coupled single-channel lead at a point $\mathbf{r}$. The system can then be characterized by the Wigner delay time $t_{W}$ (energy derivative of the scattering phase shift), whose statistical properties attracted a lot of research interest in recent years; see [8,9]. For convenience, we will consider below the dimensionless delay time $\tilde{t}_{W}=t_{W} \Delta / 2 \pi$ normalized to the mean level spacing $\Delta$. At the Anderson transition point the corresponding distribution function, $\mathcal{P}_{W}\left(\tilde{t}_{W}\right)$, will reflect the criticality of the system $[9,10]$.

To establish a connection between the MF of wave functions and that of delay times, we recall a relation between $\mathcal{P}_{W}$ and the distribution function $\mathcal{P}_{y}$ of normalized wave-function intensities $y=V\left|\psi^{2}(\mathbf{r})\right|\left(V \sim L^{d}\right.$ is the system volume),

$$
\mathcal{P}_{W}\left(\tilde{t}_{W}\right)=\tilde{t}_{W}^{-3} \mathcal{P}_{y}\left(\tilde{t}_{W}^{-1}\right) .
$$

This formula was derived in Ref. [9] and has the same status as Eq. (1): it is exact on the level of the NL $\sigma \mathrm{M}$. Equation (6) implies a relation between the exponents [9]

$$
\gamma_{q}=\tau_{1+q}
$$

where the indices $\gamma_{q}$ characterize the scaling of moments of the inverse delay time, $\left\langle t_{W}^{-q}\right\rangle \propto L^{-\gamma_{q}}$. Applying the same argumentation as used above for derivation of Eq. (2), we conclude that the relation (7) must again be exact for any generic microscopic model.

The following point should be emphasized here. Strictly speaking, the moments $\left\langle t_{W}^{-q}\right\rangle$ with $q<-3 / 2$ are divergent for the one-channel scattering problem. To define the exponent $\gamma_{q}$ for this case one should consider a lead with several conducting channels. This is analogous to the coarse-graining procedure for defining the wave-function exponent $\tau_{q}$ with negative $q$ discussed below. Equation (7) holds for such negative $q<-3 / 2$ as well, by analytical continuation.

We turn now to the analysis of consequences and applications of the derived relations, mainly concentrating on Eq. (2). First, we rewrite the relation (2) in terms of the exponents $\tau_{q}$,

$$
\tau_{q}-\tau_{1-q}=d(2 q-1) .
$$

Second, performing the Legendre transformation, $f\left(\alpha_{q}\right)=$ $q \alpha_{q}-\tau_{q}$ with $\alpha_{q}=d \tau_{q} / d q$, we get

$$
f(2 d-\alpha)=f(\alpha)+d-\alpha .
$$

Equation (9) maps the part of the singularity spectrum with $\alpha<d$ to that with $\alpha>d$. A particular consequence of this is that the support of the singularity spectrum $f(\alpha)$ (i.e., the region where it is different from $-\infty$ ) is bounded by the interval $[0,2 d]$. The lower boundary, $\alpha \geq 0$, is a trivial consequence of the wave-function normalization; the upper boundary, $\alpha \leq 2 d$, follows then from our relation (9).

It is worth mentioning that the results for the $f(\alpha)$ spectrum, as obtained numerically for the $3 d$ Anderson transition in a number of works $[11,12]$, are in conflict with this upper boundary. We believe that this is a consequence of an incomplete analysis of numerical data in [11,12]. Indeed, it was shown recently [13] that the earlier numerics on the wave-function MF suffered strongly from the absence of ensemble averaging and from finite-size effects. The problems become even more severe for negative moments, $q<0$, corresponding to the large- $\alpha$ part of the singularity spectrum. This is evident, in particular, from Fig. 6 of Ref. [12] where a strong drift of large- $\alpha$ part of $f(\alpha)$ (towards our upper boundary $\alpha \leq 2 d=6$ ) with increasing system size is seen.

Let us analyze the implication of our relation for the weak-coupling expansion of the critical exponents in $2+\epsilon$ dimensions (where MF is weak). Since Eq. (2) is exact, it should hold in all orders of the $\epsilon$ expansion. The known results for the $\epsilon$ expansion of $\Delta_{q}$ up to 4-loop order [14] do satisfy this property. In particular, the result for the orthogonal symmetry class reads

$$
\Delta_{q}=q(1-q) \epsilon+\frac{\zeta(3)}{4} q(q-1)[q(q-1)+1] \epsilon^{4}+O\left(\epsilon^{5}\right) .
$$

It is indeed seen that $\Delta_{q}$ depends on $q$ via the combination $q(1-q)$ only, in agreement with the relation (2).

In view of its exactness, Eq. (2) holds also at strongcoupling Anderson critical points, in particular, at the quantum Hall transition. Clearly, the parabolic MF spectrum, $\Delta_{q}=(\eta / 2) q(1-q)$ suggested by recent analytical proposals and numerical work [see [13] and references therein; $\eta \simeq 0.524$ ] does satisfy the relation (2) [15].

As a further application of Eq. (2), we consider the model of power-law random banded matrices (PRBM), $\left\langle\left|H_{i j}\right|^{2}\right\rangle=\left(1+|i-j|^{2} / b^{2}\right)^{-1}$. This model (that describes a $1 d$ system with long-range $1 / r$ random hopping) defines a family of critical theories parametrized by $0<b<\infty$ and allows to study the evolution of the critical system from the weak- to the strong-MF regime with decreasing $b$ $[16,17]$. In the last few years, it has attracted considerable interest as a model of the Anderson critical point [18]. While for $b \gg 1$ (weak MF) the PRBM model can be approximately mapped to the $\mathrm{NL} \sigma \mathrm{M}$, for small $b$ (strong MF) this mapping is not applicable, and the multifractal spectrum was analyzed in [17] by a different method.

Since we have a line of fixed points now, the simple universality argument (as above) is not sufficient. Nevertheless, our statement about the exactness of Eqs. (2) and (7) remains valid for the PRBM model. Indeed, we can 
construct a "granular" generalization of the model with $N \gg 1$ states at each site of the $1 d$ lattice and with hopping matrix elements between all states decaying with distance $r$ as $(\tilde{b} / N) r^{-1}$. Changing the overall prefactor $\tilde{b}$ in the hopping amplitude will yield a family of critical models that should flow in the infrared limit to the same line of fixed points as the family of PRBM models. In this way, the PRBM model with an arbitrary value of $b$ can be associated with an $N$-orbital model with some $\tilde{b}$ that will have the same critical properties. Since the latter model can be mapped to the $\mathrm{NL} \sigma \mathrm{M}$, we can again derive the relations (2) and (7) for the critical exponents.

We have verified the validity of the relation (2) by a numerical simulation of the PRBM model. The exponents $\tau_{q}$ were extracted from the scaling of the inverse participation ratios $\left\langle P_{q}\right\rangle$ for system sizes $L$ in the range from 512 to 4096 . The number of disorder realizations was ranging from $2 \times 10^{5}$ for $L=512$ to 1000 for $L=4096$. It should be stressed that evaluation of negative moments requires special care, since the inverse participation ratio, as defined in Eq. (3), is divergent because of zeros of the wave function. These zeros, related to oscillations of the wave function on the scale of the wave length, have nothing to do with multifractal properties characterizing smooth envelopes of wave functions. To find $\tau_{q}$ with negative $q$, we have first smoothed $\left|\psi^{2}\right|$ by averaging over blocks of the size $m=16$, and then applied Eq. (3). This makes finitesize effects (and thus numerical errors in $\tau_{q}$ ) for $q<0$ considerably more pronounced than for $q>0$.

The results of the numerical simulations for the PRBM ensemble with several values of $b$, spanning the whole interval from the weak-MF to strong-MF regime, are shown in Figs. 1 and 2. The data in Fig. 1 nicely confirm the symmetry relation (2). A small difference between $\Delta_{q}$ and $\Delta_{1-q}$ can be considered as a measure of the numerical accuracy of evaluation of the exponents. As discussed above, the errors are mainly due to moments with negative $q$. In Fig. 2 the same numerical data are presented in the

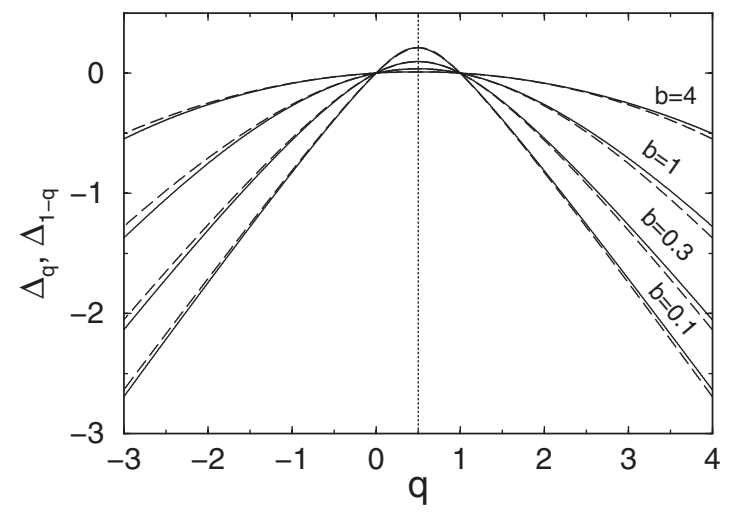

FIG. 1. Multifractal exponents $\Delta_{q}$ for the PRBM model with $b=4,1,0.3,0.1$. The symmetry (2) with respect to the point $q=1 / 2$ is evident. A small difference between $\Delta_{q}$ (full line) and $\Delta_{1-q}$ (dashed line) is due to numerical errors. form of the singularity spectrum $f(\alpha)$. To demonstrate that the data support very well the relation (9), we also show the function $f(2-\alpha)+\alpha-1$.

We will now demonstrate the high utility of Eq. (2) by applying it for the analytical evaluation of exponents with $q<1 / 2$ in the "non-NL $\sigma \mathrm{M}$ " limit, $b \ll 1$. As was found in [17], the multifractal exponents in this regime are given for $q>1 / 2$ by

$$
\tau_{q} \simeq 2 b T(q), \quad T(q)=\frac{2}{\sqrt{\pi}} \frac{\Gamma(q-1 / 2)}{\Gamma(q-1)},
$$

with $T(q)$ having the asymptotics $T(q) \simeq-1 / \pi\left(q-\frac{1}{2}\right)$ as $q \rightarrow 1 / 2$ and $T(q) \simeq(2 / \sqrt{\pi}) q^{1 / 2}$ at $q \gg 1$. In terms of the singularity spectrum $f(\alpha)$, this means

$$
f(\alpha) \simeq 2 b F(\alpha / 2 b)
$$

where $F(A)$ is the Legendre transform of $T(q)$ with

$$
F(A) \simeq \begin{cases}-1 / \pi A, & A \rightarrow 0, \\ A / 2, & A \rightarrow \infty .\end{cases}
$$

Equation (11) was derived in [17] by a real-space renormalization-group method valid for $q>1 / 2$. The relation (2) allows us now to find the multifractality spectrum for $q<1 / 2$. When translated to $f(\alpha)$ language, Eq. (9), this yields the singularity spectrum for $\alpha>1$,

$$
\begin{aligned}
f(\alpha) & \simeq 2 b F\left(\frac{2-\alpha}{2 b}\right)+\alpha-1 \\
& \simeq \begin{cases}\alpha / 2, & 2-\alpha \gg 2 b, \\
1-\frac{4 b^{2}}{\pi(2-\alpha)}, & 2-\alpha \ll 2 b .\end{cases}
\end{aligned}
$$

In Fig. 3 we show the MF spectrum of the PRBM model for $b=0.1$. The dashed curve yields the $\alpha<1$ behavior, Eq. (12), while the full line is the $\alpha>1$ result, Eq. (14).

In the limit $b \rightarrow 0$ the MF reaches its extreme form (for the PRBM model the effective spatial dimensionality $d=$ 1 ; we keep $d$ below for generality)

$$
\tau_{q}= \begin{cases}0, & q \geq 1 / 2 \\ d(2 q-1), & q \leq 1 / 2\end{cases}
$$

or, in terms of the singularity spectrum,

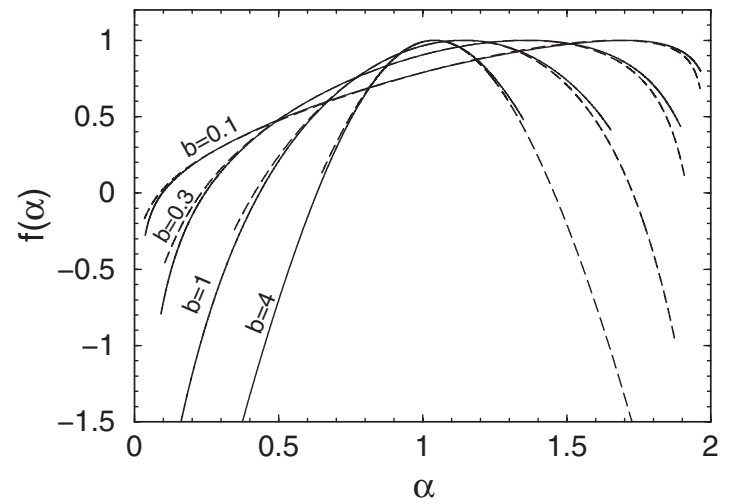

FIG. 2. The data of Fig. 1 in terms of the singularity spectrum $f(\alpha)$. Dashed lines represent $f(2-\alpha)+\alpha-1$, demonstrating the validity of Eq. (9). 


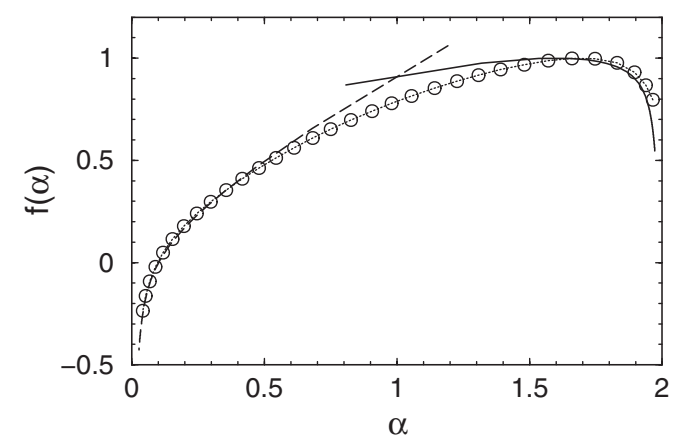

FIG. 3. Singularity spectrum for the PRBM model with $b=$ 0.1. Dashed line: $\alpha<1$ behavior, Eq. (12); full line: $\alpha>1$ result, Eq. (14), following from the relation (9); circles: numerical data. Some mismatch between the slopes of the two curves at $\alpha=1$ is related to the fact that the formula (12) is valid to the leading order in $b \ll 1$.

$$
f(\alpha)= \begin{cases}\alpha / 2, & 0 \leq \alpha \leq 2 d \\ -\infty, & \text { otherwise }\end{cases}
$$

The following remark is in order here. The earlier analysis $[2,19]$ of the statistics of critical wave functions on the Bethe lattice and in large dimensionality $d$ allows us to conjecture that in the limit $d \rightarrow \infty$ the multifractal spectrum at the Anderson transition acquires the same extreme form (15) and (16). We stress, however, that this is only a hypothesis waiting for a more rigorous verification.

The second relation we claim to be exact, Eq. (7), is also supported by numerical results obtained for the PRBM model. Specifically, the numerical data [10] for the scaling of the delay time moments confirm [in combination with the results of Ref. [17] on the wave-function MF] the validity of Eq. (7) even in the small- $b$ limit where the mapping of the PRBM to the NL $\sigma \mathrm{M}$ fails.

As a final remark, we note that the notion of MF was recently extended to the surface of a critical system [20]. While boundary multifractal exponents are different from their bulk counterparts, the relations (2) and (7) remain valid also for surface MF. Indeed, it is not difficult to check that the derivation of the relations for the distribution functions, (1) and (6), retain its validity independently on the position of the observation point $\mathbf{r}$. The MF of delay times for a lead attached to the boundary has in fact been studied numerically in the PRBM model in Ref. [10]; an analysis of the surface MF of wave functions and the verification of the relation (7) at the boundary of this system will be presented elsewhere.

To summarize, we have demonstrated that two exact relations, Eqs. (2) and (7), hold for multifractal exponents at the critical point of the Anderson transition. We have applied the first of these relations to the multifractality spectrum of the PRBM model and verified its validity by numerical simulations. A further analysis of implications of these relations is of considerable interest. Another direction is to study whether these relations, derived here for three Wigner-Dyson classes, have some analogues for unconventional symmetry classes [21].

We thank I. Gruzberg for useful discussions. This work was supported by the SPP "Quanten-Hall-Systeme" and the Center for Functional Nanostructures of the DFG (A.D.M., F. E.), and by EPSRC Grant No. EP/C515056/ 1 (Y.F.).

*Also at Petersburg Nuclear Physics Institute, 188350 St. Petersburg, Russia.

[1] M. Janssen, Phys. Rep. 295, 1 (1998); A. D. Mirlin, Phys. Rep. 326, 259 (2000).

[2] A. D. Mirlin and Y. V. Fyodorov, Phys. Rev. Lett. 72, 526 (1994); J. Phys. I (France) 4, 655 (1994).

[3] Y. V. Fyodorov and D. V. Savin, JETP Lett. 80, 725 (2004).

[4] D. V. Savin, Y. V. Fyodorov, and H.-J. Sommers, JETP Lett. 82, 544 (2005); Y. V. Fyodorov, D. V. Savin, and H.-J. Sommers, J. Phys. A 38, 10731 (2005).

[5] F. Wegner, Phys. Rep. 67, 15 (1980).

[6] K. B. Efetov, Adv. Phys. 32, 53 (1983); Supersymmetry in Disorder and Chaos (Cambridge University Press, Cambridge, England, 1997).

[7] L. P. Kadanoff, Statistical Physics: Statics, Dynamics and Renormalization (World Scientific, Singapore, 2000), Chap. IV.

[8] Y. V. Fyodorov and H.-J. Sommers, J. Math. Phys. (N.Y.) 38, 1918 (1997); T. Kottos, J. Phys. A 38, 10761 (2005).

[9] A. Ossipov and Y. V. Fyodorov, Phys. Rev. B 71, 125133 (2005).

[10] J. A. Mendez-Bermudez and T. Kottos, Phys. Rev. B 72, 064108 (2005).

[11] M. Schreiber and H. Grussbach, Phys. Rev. Lett. 67, 607 (1991); H. Grussbach and M. Schreiber, Phys. Rev. B 51, 663 (1995); T. Terao, Phys. Rev. B 56, 975 (1997).

[12] M. Schreiber, F. Milde, and R. A. Römer, Comput. Phys. Commun. 121-122, 517 (1999).

[13] F. Evers, A. Mildenberger, and A. D. Mirlin, Phys. Rev. B 64, 241303(R) (2001).

[14] F. Wegner, Nucl. Phys. B280, 210 (1987).

[15] The parabolic behavior should terminate on both sides at $|q-1 / 2|=2 / \eta$. The upper cutoff is due to $\alpha \geq 0$; the negative- $q$ cutoff follows from our symmetry relation.

[16] A. D. Mirlin et al., Phys. Rev. E 54, 3221 (1996).

[17] A. D. Mirlin and F. Evers, Phys. Rev. B 62, 7920 (2000).

[18] E. Cuevas et al., Phys. Rev. Lett. 88, 016401 (2001); I. Varga, Phys. Rev. B 66, 094201 (2002); E. Cuevas, Phys. Rev. B 68, 024206 (2003); 68, 184206 (2003); 71, 024205 (2005); O. Yevtushenko and V.E. Kravtsov, Phys. Rev. E 69, 026104 (2004); V. E. Kravtsov, O. Yevtushenko, and E. Cuevas, J. Phys. A 39, 2021 (2006).

[19] A. Mildenberger, F. Evers, and A. D. Mirlin, Phys. Rev. B 66, 033109 (2002).

[20] A. R. Subramaniam et al., Phys. Rev. Lett. 96, 126802 (2006).

[21] A. Altland and M. R. Zirnbauer, Phys. Rev. B 55, 1142 (1997); M. R. Zirnbauer, J. Math. Phys. (N.Y.) 38, 2007 (1997). 